\title{
Andreev-Coulomb Drag in Coupled Quantum Dots
}

\author{
S. Mojtaba Tabatabaei®, ${ }^{1}$ David Sánchez $\odot,{ }^{2}$ Alfredo Levy Yeyati $\odot,{ }^{3}$ and Rafael Sánchez $\odot^{3}$ \\ ${ }^{1}$ Department of Physics, Shahid Beheshti University, G. C. Evin, 1983963113 Tehran, Iran \\ ${ }^{2}$ Institute for Cross-Disciplinary Physics and Complex Systems IFISC (UIB-CSIC), E-07122 Palma de Mallorca, Spain \\ ${ }^{3}$ Departamento de Física Teórica de la Materia Condensada, Condensed Matter Physics Center (IFIMAC), and \\ Instituto Nicolás Cabrera, Universidad Autónoma de Madrid, 28049 Madrid, Spain
}

(Received 11 August 2020; accepted 29 October 2020; published 7 December 2020)

\begin{abstract}
The Coulomb drag effect has been observed as a tiny current induced by both electron-hole asymmetry and interactions in normal coupled quantum dot devices. In the present work we show that the effect can be boosted by replacing one of the normal electrodes by a superconducting one. Moreover, we show that at low temperatures and for sufficiently strong coupling to the superconducting lead, the Coulomb drag is dominated by Andreev processes, is robust against details of the system parameters, and can be controlled with a single gate voltage. This mechanism can be distinguished from single-particle contributions by a sign inversion of the drag current.
\end{abstract}

DOI: 10.1103/PhysRevLett.125.247701

Introduction.-The possibility to induce a current in an unbiased electronic circuit by proximity with a nearby driven system is a measurable manifestation of electronelectron correlations and broken symmetries. For this reason, the Coulomb drag effect has attracted theoretical and experimental attention for many decades [1]. In extended samples with translational invariance, momentum exchange across conductors is the relevant drag mechanism [2]. Remarkably, when the samples are superconducting (e.g., Coulomb coupled bilayers or Josephson junction arrays) Cooper pairs must be taken into account $[3,4]$, leading to drag supercurrents [5], charge solitons [6], exciton formation [7], and depinning [8] (see also Ref. [9] for a discussion of drag currents in normal tunnel junction arrays).

In contrast, in mesoscopic systems such as coupled quantum dots or point contacts, which lack translational invariance, Coulomb drag is driven by energy transfer. Moreover, finite drag currents with normal leads require the presence of energy-dependent tunneling rates breaking electron-hole and inversion symmetries [10-19]. Although this leads to a rather tiny and uncontrolled effect strongly dependent on the system parameters, drag currents have been observed in nanoelectronic devices [20-24]. The mesoscopic Coulomb drag can also be viewed as arising from rectifying nonequilibrium charge fluctuations. This makes the connection with exciting issues in modern quantum thermodynamics: energy harvesting [25-27], heat transport [28-30], and rectifiers [31,32].

In this Letter, we explore how the mesoscopic Coulomb drag is affected by superconducting correlations. Let us consider a proximitized nanostructure [33] like the one depicted in Fig. 1(a). It consists of two capacitively coupled quantum dots [34-36]: the active dot is coupled to two voltage-biased normal reservoirs, whereas the passive one is coupled to a normal and a superconducting lead (hence breaking inversion symmetry). Because of the presence of a superconducting gap, the tunneling rates exhibit a well defined energy dependence which has proven useful for quasiparticle turnstiles [37] and refrigeration [38-41].

(a)
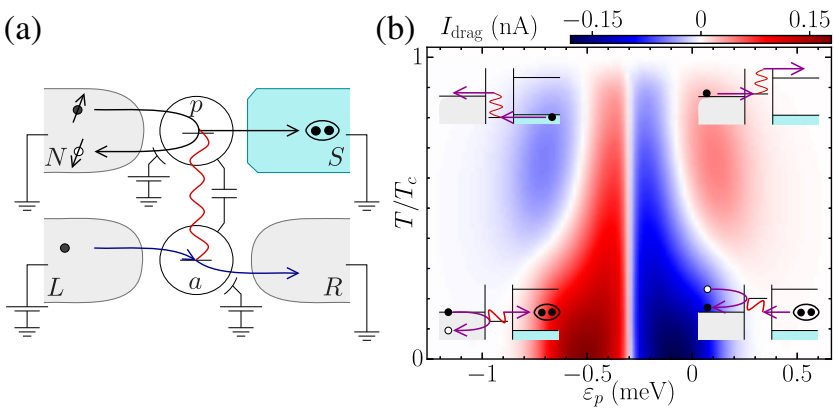

FIG. 1. Superconductivity-induced Coulomb drag in hybrid dot structures. (a) Scheme of the capacitively coupled double quantum dot device analyzed in this work. A drag current is generated in the passive dot $(p)$ connected to unbiased normal $(N)$ and superconducting $(S)$ electrodes due to the Coulomb interaction with electrons tunneling through the active dot $a$ attached to voltage biased terminals ( $L$ and $R$ ). In this diagram, an electron in $N$ is transformed into a Cooper pair in $S$ with a retroreflected hole. This Andreev process is correlated through the interaction (red line) with a charge fluctuation in the active subsystem (blue arrow). (b) Drag current $I_{\text {drag }}$ as a function of the passive dot level $\varepsilon_{p}$ and temperature $T$ (normalized with the critical temperature $T_{c}$ ). Cooper pairs and quasiparticles contribute with opposite signs. At low temperature, the current is given by Andreev processes (lower insets). By increasing temperature, a crossover occurs where they coexist with quasiparticle tunneling (upper insets). Parameters (in meV): $\Delta(T=0)=0.2, \quad \Gamma_{S}=\Gamma_{N}=\Gamma_{L}=$ $\Gamma_{R}=0.05, V_{\text {bias }}=5, \varepsilon_{a}=0$, and $U_{p}=5 U_{a p}=0.5$. 
Superconductivity gives rise, however, to an additional and totally different drag mechanism via the Andreev reflection of an electron into a hole at the passive quantum dot [42]. Mediated by Coulomb interactions, charge fluctuations in the active dot correlate with the pairing processes, hence breaking electron-hole symmetry, as schematically depicted in the lower insets of Fig. 1(b). A key role is played by the coherent superposition of the passive dot charge states induced by pairing and which can be controlled by external gate voltages. Tuning the position of the passive dot level, the drag current would display a transition from electronlike to holelike dominated behavior. As we show in this work, the Andreev drag mechanism dominates at low temperature $T$ for realistic system parameters, and could be distinguished from the conventional (single particle) processes by a drag current sign reversal in state-of-the-art experiments.

Model and methods.-We model the device depicted in Fig. 1(a) with a Hamiltonian of the form $\hat{H}=$ $\hat{H}_{\text {dqd }}+\hat{H}_{\text {leads }}+\hat{H}_{t}$, where

$$
\hat{H}_{\mathrm{dqd}}=\sum_{\alpha} \varepsilon_{\alpha} \hat{n}_{\alpha}+U_{p} \hat{n}_{p, \uparrow} \hat{n}_{p, \downarrow}+U_{a p} \hat{n}_{a} \hat{n}_{p},
$$

is the double quantum dot Hamiltonian with active $(\alpha=a)$ and passive $(\alpha=p)$ dots, energy levels $\varepsilon_{\alpha}$, intradot $\left(U_{p}\right)$ and interdot $\left(U_{a p}\right)$ charging energies and number operators $\hat{n}_{p}=\Sigma_{\sigma} \hat{n}_{p, \sigma}=\Sigma_{\sigma} \hat{d}_{p, \sigma}^{\dagger} \hat{d}_{p, \sigma}$ with spin $\sigma=\{\uparrow, \downarrow\} \quad$ and $\hat{n}_{a}=\hat{d}_{a}^{\dagger} \hat{d}_{a}$, where $\hat{d}_{p, \sigma}$ and $\hat{d}_{a}$ are the electron annihilation operators in passive and active dots (note that, for simplicity, the active dot is described in terms of spinless electrons as this degree of freedom does not play a role in the drag physics). $\hat{H}_{\text {leads }}$ and $\hat{H}_{t}$ are the uncoupled leads and the lead-dot tunneling Hamiltonians, respectively. While the passive dot is coupled to a normal $(N)$ and a superconducting $(S)$ lead, the active dot is connected to two normal ones ( $L$ and $R$ ). The corresponding tunneling rates are denoted by $\Gamma_{\beta}$, where $\beta=N, S, L, R$ labels the terminals with chemical potential $\mu_{\beta}$. Since the passive dot is in equilibrium we set $\mu_{N}=\mu_{S}=0$, while the active dot is voltage biased as $\mu_{L}=-\mu_{R}=e V_{\text {bias }} / 2$.

In order to obtain the transport properties for this model we apply two complementary approaches. For the numerical calculations, we use a nonequilibrium Green's functions (NEGF) formalism including up to second-order diagrams for the interaction self-energies and taking into account the BCS temperature dependence for the $S$ lead gap. Additionally, we use a master equation description to analytically identify the relevant processes responsible for the drag current. This latter approach is valid in two limiting cases: (i) the drag is carried by Cooper pairs (assuming the infinite limit for the superconducting gap $\Delta$ ), and (ii) pairing is neglected and drag is due to singlequasiparticle processes only [43].
The drag current as a function of temperature and passive dot level obtained using NEGF is shown in Fig. 1(b). The chosen parameters correspond to realistic values that can be achieved in current experimental setups [33]. As a first remark, we notice that the drag current can reach values of the order of $0.1 \mathrm{nA}$, which can be easily detected experimentally [24]. Second, we observe four different regions depending on the sign of the drag current. At low temperatures, this sign changes when $\epsilon_{p}$ crosses the electron-hole symmetric point $\varepsilon_{p}^{\mathrm{eh}}=-\left(U_{p}+U_{a p}\right) / 2$, turning from positive to negative as the passive dot is depopulated. At higher temperatures an additional sign change occurs, while the size of the drag current is significantly reduced, and eventually disappears when the gap is closed for $T>T_{c}$. As discussed below, this change of behavior is physically connected to the microscopic mechanisms yielding the Coulomb drag, pinpointing a crossover from the Andreev drag regime to the quasiparticle tunneling regime as temperature is raised.

Drag out of correlated Andreev reflection.-We can gain insight on the low temperature behavior using the master equation approach with the transition rates calculated in the limit $\Delta, e V_{\text {bias }} \gg k_{B} T$. In this approximate analysis, the relevant basis states are $|i, n\rangle$, where $i=\{0, \sigma, 2\}$ denotes the occupation of the passive dot and $n=0,1$ is the number of electrons in the active one. Pairing induced by coupling to the superconductor hybridizes the even- $i$ states in the passive dot, leading to $| \pm, n\rangle=$ $\mathcal{N}_{ \pm, n}^{-1}\left(A_{ \pm, n}|0, n\rangle-\Gamma_{S}|2, n\rangle\right)$, with $A_{ \pm, n}=\tilde{\varepsilon}_{n} \pm \sqrt{\tilde{\varepsilon}_{n}^{2}+\Gamma_{S}^{2}}$, $\tilde{\varepsilon}_{n}=\varepsilon_{p}+n U_{a p}+U_{p} / 2$, and the normalization factor $\mathcal{N}_{ \pm, n}$. The odd states $|\sigma, n\rangle$ remain uncoupled. Importantly, the even superpositions and the eigenenergies $E_{ \pm, n}=n \varepsilon_{a}+A_{\mp, n}, E_{\sigma n}=\varepsilon_{p}+n\left(\varepsilon_{a}+U_{a p}\right)$ depend on the occupation of the active dot, see Fig. 2(a).

In the weak tunneling regime, the transition rates between the different states $|\kappa\rangle \rightarrow|\lambda\rangle$ are given by $\Gamma_{\lambda \kappa}^{\alpha \beta}=$ $\mathcal{G}_{\lambda \kappa}^{\alpha \beta} f_{\beta}\left(E_{\lambda}-E_{\kappa}\right)$ when involving an electron tunneling from terminal $\beta$ into quantum dot $\alpha$, and $\gamma_{\lambda \kappa}^{\alpha \beta}=\mathcal{J}_{\lambda \kappa}^{\alpha \beta}\left[1-f_{\beta}\left(E_{\kappa}-\right.\right.$ $\left.E_{\lambda}\right)$ ] when involving a hole [53-55]. Here, $\mathcal{G}_{\lambda \kappa}^{\alpha \beta}=$ $\Gamma_{\beta}\left|\left\langle\lambda\left|\hat{\delta}_{\alpha}^{\dagger}\right| \kappa\right\rangle\right|^{2}$ are the leading-order tunneling rates with $\hat{\delta}_{a} \equiv \hat{d}_{a}, \quad \hat{\delta}_{p} \equiv \sum_{\sigma} \hat{d}_{p, \sigma}, \quad$ and $\quad f_{\beta}(E)=\{1+\exp [(E-$ $\left.\left.\left.\mu_{\beta}\right) / k_{B} T\right]\right\}^{-1}$ the Fermi-Dirac function. For $\mathcal{J}_{\lambda k}^{\alpha \beta}$, one replaces $\hat{\delta}_{\alpha}^{\dagger}$ by $\hat{\delta}_{\alpha}$ in the expression for $\mathcal{G}_{\lambda \kappa}^{\alpha \beta}$.

At low temperatures, transitions $|\kappa\rangle \rightarrow|\lambda\rangle$ involving terminal $N$ are energetically suppressed if $E_{\lambda}-E_{\kappa} \gg$ $\mu_{N}=0$. Noticing that $E_{ \pm n}-E_{\sigma n}=U_{p} / 2 \mp \sqrt{\tilde{\varepsilon}_{n}^{2}+\Gamma_{S}^{2}}$, we see that transitions to $|-, n\rangle$ are only possible if mediated by a fluctuation in the active dot. Because of the interaction $U_{p}$, the energies of the $|+, n\rangle$ and $|\sigma, n\rangle$ states might cross, as shown in Fig. 2(a). Hence, for a constant $n$ the passive dot is in a statistical mixture of states $|+, n\rangle$ and $|\sigma, n\rangle$. Then, electrons tunneling from $L$ to $R$ change the charge $n$ and consequently the passive dot 


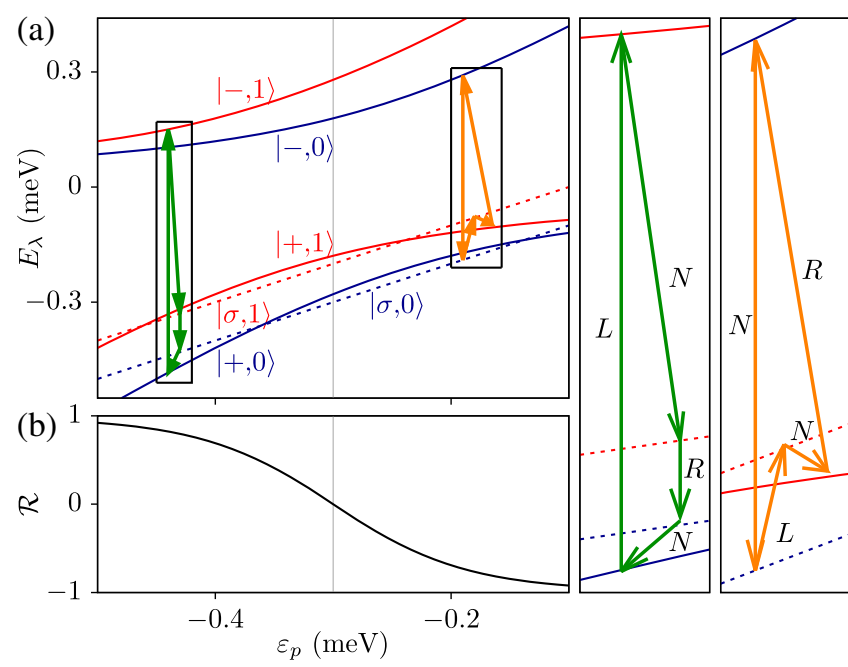

FIG. 2. Andreev-Coulomb drag mechanism. (a) Pairing induces avoided crossings in the even states $| \pm, n\rangle$. Two cyclic processes are highlighted which correspond to Eq. (2), with $n=1, n^{\prime}=0$ (orange) and $n=0, n^{\prime}=1$ (green). These cycles are assisted by charge fluctuations in the active dot and lead to finite drag currents. (b) The imbalance of tunneled electrons and holes dictates the sign of the current by means of the asymmetry of the transition rates, parametrized here by $\mathcal{R}=\left(r_{+0} r_{-1}-1\right) /$ $\left(r_{+0} r_{-1}+1\right)$, depending on the level position $\varepsilon_{p}$. The gray line in (a) and (b) marks the electron-hole symmetry point $\varepsilon_{p}^{\mathrm{eh}}$. Parameters are as in Fig. 1(b).

undergoes transitions between different states. In particular, depending on which is the ground state, the transitions $|+, n\rangle \leftrightarrow|-, n \pm 1\rangle$ and $|\sigma, n\rangle \leftrightarrow|\sigma, n \pm 1\rangle$ are important for unblocking the dynamics. Furthermore, they enable cycles of the form

$$
|\sigma, n\rangle \rightarrow|+, n\rangle \leftrightarrow\left|-, n^{\prime}\right\rangle \rightarrow\left|\sigma, n^{\prime}\right\rangle \leftrightarrow|\sigma, n\rangle,
$$

as sketched in Fig. 2(a) by the green (for $n^{\prime}=n+1$ ) and orange (for $n^{\prime}=n-1$ ) arrows. They include two transitions in terminal $N$ involving different superpositions at different occupations of the active dot, $|+, n\rangle$ and $\left|-, n^{\prime}\right\rangle$. Thus, the weights in these superpositions are changed in the processes due to both pairing and Coulomb interactions. These are the minimal cycles that allow for a net charge exchange between $N$ and $S$ and ultimately lead to a drag current.

The drag effect requires the electron-hole symmetry to be dynamically broken [13]. In our system, transitions $|\sigma, n\rangle \rightarrow|+, n\rangle$ and $|-, n\rangle \rightarrow|\sigma, n\rangle$ can both be due to the tunneling of either an electron or a hole into the passive dot. Whether electron or hole transport dominates depends in each case on the relative weight of the even states via the ratios $r_{+n}=\mathcal{G}_{+n, \sigma n}^{p N} / \mathcal{J}_{+n, \sigma n}^{p N}=\Gamma_{S}^{2} / A_{+n}^{2}$ and $r_{-n}=\mathcal{G}_{\sigma n,-n}^{p N} /$ $\mathcal{J}_{\sigma n,-n}^{p N}=A_{-n}^{2} / \Gamma_{S}^{2}$. In the cycles of Eq. (2), more electrons than holes will on average be transferred if $r_{+n} r_{-n^{\prime}}>1$. Remarkably, these ratios can be controlled by tuning the passive dot energy level with a gate voltage. For $r_{ \pm n}>1$ electron tunneling will occur at a higher rate than hole tunneling if $\tilde{\varepsilon}_{n}<0$. In this way, if $\tilde{\varepsilon}_{0}<\tilde{\varepsilon}_{1}<0$ (like the green example in Fig. 2), we have $A_{+, n}^{2}<\Gamma_{S}^{2}<A_{-, n}^{2}$. Then most likely, two electrons tunnel from $N$ into the passive dot along with a Cooper pair created in the superconductor, giving rise to a positive drag current. In the opposite case $\tilde{\varepsilon}_{0}>0$ (like for the orange cycle in Fig. 2), the two transitions are holelike and the drag current is accordingly reversed. As shown in Fig. 2(b), $r_{+n} r_{-n^{\prime}}-1$ is monotonic when tuning $\varepsilon_{p}$ across the intermediate region. It changes sign at $\varepsilon_{p}^{\mathrm{eh}}$, together with $I_{\text {drag }}$ [see Fig. 1(b)]. At this point electron and hole processes occur with the same rate during the cycle and cancel each other, resulting in no net drag current.

We stress that the pairing term is necessary for the drag current in the Andreev regime since (i) it splits the degeneracy points of even and odd states, and (ii) it breaks electron-hole symmetry by making $r_{ \pm n} \neq 1$. However, increasing the $S$ coupling has a counterproductive effect: in the limit $\Gamma_{S} \gg \tilde{\varepsilon}_{n}$ we find $r_{ \pm n} \rightarrow 1$, i.e., tunneling becomes electron-hole symmetric and the drag effect accordingly vanishes.

Note that this drag effect differs from the normal one $[13,16,17]$ in that both the existence of the drag current and its sign are determined by $\varepsilon_{p}$, while in normal systems both depend on energy-resolved tunneling asymmetries. Our device can also host such a conventional drag effect when current is carried by quasiparticles, as discussed below. Strikingly enough, the sign of their contribution is also well defined in terms of $\varepsilon_{p}$ but is opposite to that of the AndreevCoulomb drag processes. This would be useful for an experimental distinction of the two mechanisms.

Quasiparticle drag.-Let us now consider a regime for which pair tunneling can be neglected (e.g., high temperatures and quantum dot levels close to the gap edges) and therefore the number of electrons in the passive dot is well defined. In a qualitative analysis, we can restrict the charge of the passive dot to fluctuate between 0 or 1 . In the weak coupling regime, transport through the $S$ barrier consists of single-electron tunneling events whose rate $\Gamma_{S n}^{\mathrm{qp}} \propto \nu_{S}\left(\varepsilon_{p}+\right.$ $\left.n U_{a p}\right)$ has an explicit energy dependence given by the superconductor density of states $\nu_{S}(E)$ [43]. Since the rate is sensitive to the charge state of the active dot $n$ due to the Coulomb interaction $U_{a p}$, this situation is then analogous to that of Ref. [13], where the drag effect appears in the presence of energy-dependent barriers. Here, such dependence is provided by the superconducting gap, even if all other couplings $\Gamma_{\beta}$ are constant. Thus, a drag current appears proportional to $\Gamma_{S 1}^{\mathrm{qp}}-\Gamma_{S 0}^{\mathrm{qp}}$, which depends on $\varepsilon_{p}$. For instance, if $-\Delta<\varepsilon_{p}<\Delta$ and $\varepsilon_{p}+U_{a p}>\Delta$, we have $\Gamma_{S 1}^{\mathrm{qp}} \gg \Gamma_{S 0}^{\mathrm{qp}}$, and consequently electrons tunnel from $N$ when $n=0$ and into $S$ over the gap once the active dot gets occupied (thereby $n$ changing to 1 ). This causes a positive drag current. For $\varepsilon_{p}<-\Delta$ and 

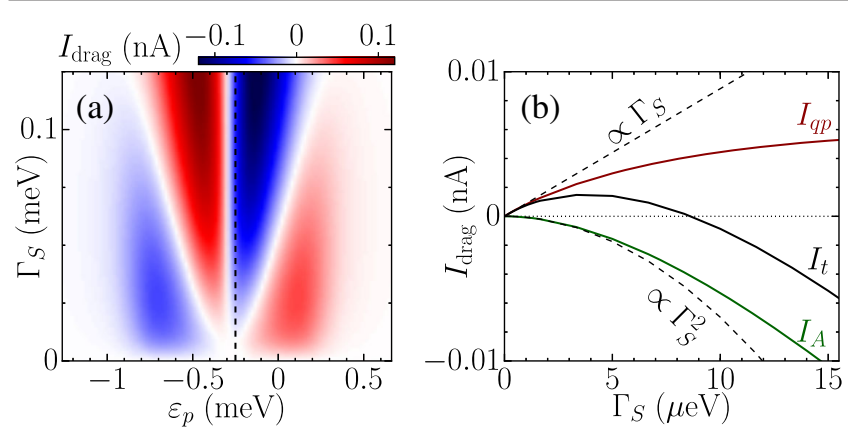

FIG. 3. Unraveling the Andreev and quasiparticle contributions to the drag current. (a) Drag current as a function of $\Gamma_{S}$ and $\varepsilon_{p}$. (b) Different contributions from the single quasiparticle $\left(I_{\mathrm{qp}}\right)$ and pair tunneling $\left(I_{A}\right)$ currents to the total drag current $\left(I_{t}\right)$. Dashed lines in (b) are fitting curves to the $\Gamma_{S}$ and $\Gamma_{S}^{2}$ lines. Parameters are as in Fig. 1, except for $T=2 T_{c} / 3$.

$-\Delta<\varepsilon_{p}+U_{a p}<\Delta$, the same happens for holes tunneling below the gap ( $n$ changing from 1 to 0 ) and the current becomes negative. Both cases are illustrated with the upper insets in Fig. 1(b). As in the case of the pairing contribution, the current changes sign at $\varepsilon_{p}=\varepsilon_{p}^{\mathrm{eh}}$.

Andreev vs quasiparticle drag.-The two limiting cases discussed above lead to opposite contributions to the drag current for the same configuration of the dot levels. Their competition will then determine the overall sign of the generated current, for instance, as a function of temperature as already shown in Fig. 1(b). The value of $\varepsilon_{p}$ relative to the crossover depends on temperature since the BCS gap changes with temperature. We furthermore investigate this competition as a function of the pairing parameter $\Gamma_{S}$ in Fig. 3. Since the leading contribution for the quasiparticle tunneling is sequential, it is of the order $\Gamma_{S} \Gamma_{N}$. However, the Andreev contribution relies on higher order processes and depends on $\Gamma_{S}$ (through $\left.r_{ \pm n}\right)$ as $\left(\Gamma_{S} \Gamma_{N}\right)^{2}$. Hence, for small values of $\Gamma_{S}$ the quasiparticle contribution dominates. As the pairing term increases, the Andreev-Coulomb mechanism starts to govern the drag effect. This is reflected in a sign change of the drag current as a function of $\Gamma_{S}$ for a fixed dot level, see Fig. 3(a).

This interpretation is confirmed by means of an energyresolved separation of the different contributions to the drag current within the NEGF calculations. Let $I_{A}$ be the subgap term, which we expect to be mainly due to Andreev processes, and $I_{\mathrm{qp}}$ the contribution over and below the gap, mainly due to quasiparticles (see Ref. [43] for further details). These are plotted in Fig. 3(b) for $\varepsilon_{p}>\varepsilon_{p}^{\mathrm{eh}}$ [along the dashed line in Fig. 3(a)]. It indeed shows that for small $\Gamma_{S}, I_{A}$ is quadratic and negative, while $I_{\mathrm{qp}}$ is linear and positive, as expected for the Andreev and quasiparticle processes discussed above.

Practical considerations. - In a typical experiment, one could tune the gate voltages that control $\varepsilon_{a}$ and $\varepsilon_{p}$, as well as the bias voltage $V_{\text {bias }}$ applied to the active dot. The drag effect manifests as a correlation between the currents
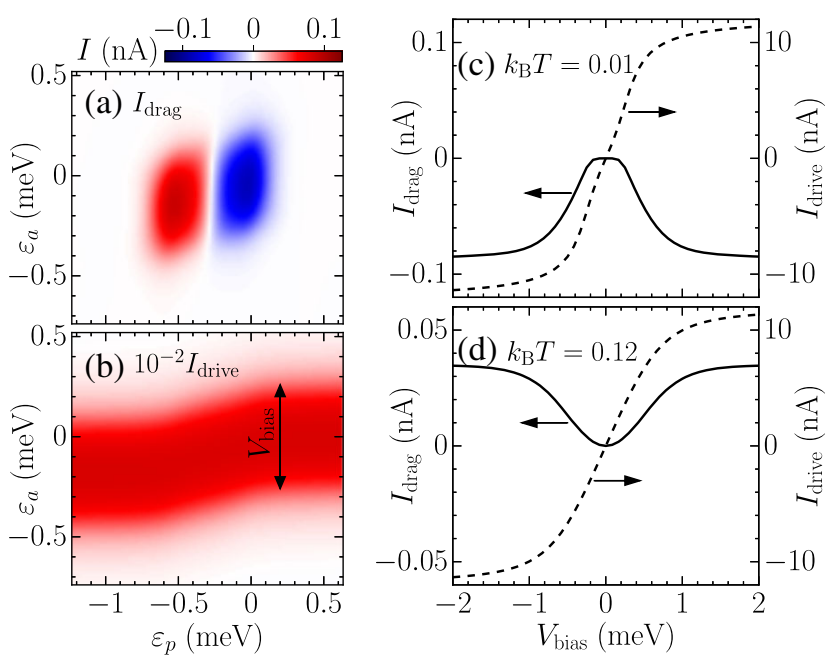

FIG. 4. Drag and drive currents as functions of experimentally tunable gate and bias voltages. (a),(b) Mutual backaction of the drag and drive systems as $\varepsilon_{a}$ and $\varepsilon_{p}$ scan the degeneracy points of the stability diagram for $k_{B} T=0.01 \mathrm{meV}$ and $V_{\text {bias }}=0.6 \mathrm{meV}$. (c),(d) The drag current does not change sign at zero bias voltage, proving its nonlinearity, here for $\varepsilon_{p}=0$. (c) At low temperatures, the Andreev-Coulomb drag current is blocked at low voltages. Lifting of the blockade affects the drive response. (d) At higher temperatures, these features are washed out. Quasiparticle contributions change the sign of the current. Other parameters are as in Fig. 1 and $k_{B} T_{c}=0.15 \mathrm{meV}$.

through the active and passive dots, see Figs. 4(a) and 4(b). These correlations induced by $U_{a p}$ are enhanced in the vicinity of the charge stability diagram degeneracy points, which give rise to an upward shift in the drive current, cf. Fig. 4(b). This behavior is typical of fluctuationinduced transport in normal double dot systems and leads to a current spot in the passive system whose sign depends on the potential in the passive system [23,24,26]. Remarkably, for the Andreev-Coulomb drag effect discussed here the induced current feature is split into electronlike and holelike contributions with opposite signs, as shown in Fig. 4(a).

The dependence on the bias voltage plotted in Figs. 4(c) and 4(d) shows that the drag current appears at the onset of nonlinearities in the drive current for large enough voltages, as expected for active systems with no energy-dependent tunneling rates $[13,17]$. This is particularly clear at low temperatures where $I_{\text {drag }}$ is blocked for low voltages, see Fig. 4(c). The size of the blockade region scales linearly with the Coulomb interaction $U_{a p}$. At higher temperatures [Fig. 4(d)] the blockade is smeared out. Further, we observe that the drag current changes sign with temperature due to the dominance of either pair-correlated [Fig. 4(c)] or singleelectron [Fig. 4(d)] processes. This behavior is absent in coupled superconducting wires or layers when their drag effect is due to supercurrents [5]. In Josephson junction arrays, the drag current can change its sign when the superconducting gap is suppressed $[6,8]$. However, in these 
devices the induced current flows in the same (opposite) direction as the drive current when transport is due to pairs (quasiparticles), while in our case it is independent of the direction of the drive and can be controlled by a local gate electrode.

Conclusions.-We have shown that pair tunneling in interacting quantum dots coupled to normal and superconducting electrodes leads to a novel drag mechanism. This Andreev-Coulomb drag can be distinguished from the conventional single-electron mechanism by means of a sign change in the drag current. Using two different theoretical methods, we have evaluated the drag currents showing that the Andreev mechanism is a robust effect and dominates at sufficiently small temperatures and strong coupling to the superconducting electrode. Our estimations, based on realistic parameters, indicate that the effect would be significantly strong to be detected using nowadays experimental techniques.

Work funded by MICINN (Ministerio de Ciencia e Innovación) under Grants No. MAT2017-82639, No. RYC2016-20778, No. FIS2017-84860-R, and No. PID2019-110125GB-I00, and the María de Maeztu Programme for Units of Excellence in R\&D (Grants No. MDM2017-0711 and No. CEX2018-000805-M).

[1] B. N. Narozhny and A. Levchenko, Coulomb drag, Rev. Mod. Phys. 88, 025003 (2016).

[2] A. G. Rojo, Electron-drag effects in coupled electron systems, J. Phys. Condens. Matter 11, R31 (1999).

[3] X. Huang, G. Bazan, and G. H. Bernstain, Observation of Supercurrent Drag between Normal Metal and Superconducting Films, Phys. Rev. Lett. 74, 4051 (1995).

[4] A. Kamenev and Y. Oreg, Coulomb drag in normal metals and superconductors: Diagrammatic approach, Phys. Rev. B 52, 7516 (1995).

[5] J.-M. Duan and S. Yip, Supercurrent Drag via the Coulomb Interaction, Phys. Rev. Lett. 70, 3647 (1993).

[6] H. Shimada, C. Ishida, and Y. Mizugaki, Drag Current Reversal in Capacitively Coupled Arrays of Small Josephson Junctions, Phys. Rev. Lett. 109, 196801 (2012).

[7] D. Bercioux, T. M. Klapwijk, and F. S. Bergeret, Transport Properties of an Electron-Hole Bilayer in Contact with a Superconductor Hybrid Junction, Phys. Rev. Lett. 119, 067001 (2017).

[8] S. A. Wilkinson, N. Vogt, and J. H. Cole, Coulomb drag and depinning in bilinear Josephson junction arrays, New J. Phys. 19, 093023 (2017).

[9] D. V. Averin, A. N. Korotkov, and Yu. V. Nazarov, Transport of Electron-Hole Pairs in Arrays of Small Tunnel Junctions, Phys. Rev. Lett. 66, 2818 (1991).

[10] N. A. Mortensen, K. Flensberg, and A.-P. Jauho, Coulomb Drag in Coherent Mesoscopic Systems, Phys. Rev. Lett. 86, 1841 (2001).

[11] A. Levchenko and A. Kamenev, Coulomb Drag in Quantum Circuits, Phys. Rev. Lett. 101, 216806 (2008).
[12] V. Moldoveanu and B. Tanatar, Coulomb drag in parallel quantum dots, Europhys. Lett. 86, 67004 (2009).

[13] R. Sánchez, R. López, D. Sánchez, and M. Büttiker, Mesoscopic Coulomb Drag, Broken Detailed Balance, and Fluctuation Relations, Phys. Rev. Lett. 104, 076801 (2010).

[14] R. Hussein and S. Kohler, Coherent quantum ratchets driven by tunnel oscillations: Fluctuations and correlations, Phys. Rev. B 86, 115452 (2012).

[15] R. Hussein and S. Kohler, Capacitively coupled nano conductors, Ann. Phys. (Berlin) 527, 610 (2015).

[16] K. Kaasbjerg and A.-P. Jauho, Correlated Coulomb Drag in Capacitively Coupled Quantum-Dot Structures, Phys. Rev. Lett. 116, 196801 (2016).

[17] M. A. Sierra, D. Sánchez, A.-P. Jauho, and K. Kaasbjerg, Fluctuation-driven Coulomb drag in interacting quantum dot systems, Phys. Rev. B 100, 081404(R) (2019).

[18] C. Zhou and H. Guo, Coulomb drag between quantum wires: A nonequilibrium many-body approach, Phys. Rev. B 99, 035423 (2019).

[19] W.-X. He, Z. Cao, G.-Y. Li, L. Li, H.-F. Lü, Z. Li, and H.-G. Luo, Performance of the $T$-matrix based master equation for Coulomb drag in double quantum dots, Phys. Rev. B 101, 035417 (2020).

[20] G. Shinkai, T. Hayashi, T. Ota, K. Muraki, and T. Fujisawa, Bidirectional current drag induced by two-electron cotunneling in coupled double quantum dots, Appl. Phys. Express 2, 081101 (2009).

[21] D. Laroche, G. Gervais, M. P. Lilly, and J. L. Reno, Positive and negative Coulomb drag in vertically integrated onedimensional quantum wires, Nat. Nanotechnol. 6, 793 (2011).

[22] D. Laroche, G. Gervais, M. P. Lilly, and J. L. Reno, 1D-1D Coulomb drag signature of a Luttinger liquid, Science 343, 631 (2014).

[23] D. Bischoff, M. Eich, O. Zilberberg, C. Rössler, T. Ihn, and K. Ensslin, Measurement back-action in stacked graphene quantum dots, Nano Lett. 15, 6003 (2015).

[24] A. J. Keller, J. S. Lim, D. Sánchez, R. López, S. Amasha, J. A. Katine, H. Shtrikman, and D. Goldhaber-Gordon, Cotunneling Drag Effect in Coulomb-Coupled Quantum Dots, Phys. Rev. Lett. 117, 066602 (2016).

[25] R. Sánchez and M. Büttiker, Optimal energy quanta to current conversion, Phys. Rev. B 83, 085428 (2011).

[26] H. Thierschmann, R. Sánchez, B. Sothmann, F. Arnold, C. Heyn, W. Hansen, H. Buhmann, and L. W. Molenkamp, Three-terminal energy harvester with coupled quantum dots, Nat. Nanotechnol. 10, 854 (2015).

[27] B. Roche, P. Roulleau, T. Jullien, Y. Jompol, I. Farrer, D. A. Ritchie, and D. C. Glattli, Harvesting dissipated energy with a mesoscopic ratchet, Nat. Commun. 6, 6738 (2015).

[28] J. V. Koski, A. Kutvonen, I. M. Khaymovich, T. Ala-Nissila, and J. P. Pekola, On-Chip Maxwells Demon as an Information-Powered Refrigerator, Phys. Rev. Lett. 115, 260602 (2015).

[29] R. Sánchez, H. Thierschmann, and L. W. Molenkamp, Allthermal transistor based on stochastic switching, Phys. Rev. B 95, 241401(R) (2017).

[30] B. Bhandari, G. Chiriacò, P. A. Erdman, R. Fazio, and F. Taddei, Thermal drag in electronic conductors, Phys. Rev. B 98, 035415 (2018). 
[31] V. S. Khrapai, S. Ludwig, J. P. Kotthaus, H. P. Tranitz, and W. Wegscheider, Double-Dot Quantum Ratchet Driven by an Independently Biased Quantum Point Contact, Phys. Rev. Lett. 97, 176803 (2006).

[32] F. Hartmann, P. Pfeffer, S. Höfling, M. Kamp, and L. Worschech, Voltage Fluctuation to Current Converter with Coulomb-Coupled Quantum Dots, Phys. Rev. Lett. 114, 146805 (2015).

[33] S. D. Franceschi, L. Kouwenhovem, C. Schönenberger, and W. Wernsdofer, Hybrid superconductor-quantum dot devices, Nat. Nanotechnol. 5, 703 (2010).

[34] I. H. Chan, R. M. Westervelt, K. D. Maranowski, and A. C. Gossard, Strongly capacitively coupled quantum dots, Appl. Phys. Lett. 80, 1818 (2002).

[35] A. Hübel, J. Weis, W. Dietsche, and K. v. Klitzing, Two laterally arranged quantum dot systems with strong capacitive interdot coupling, Appl. Phys. Lett. 91, 102101 (2007).

[36] D. T. McClure, L. DiCarlo, Y. Zhang, H.-A. Engel, C. M. Marcus, M. P. Hanson, and A. C. Gossard, Tunable Noise Cross Correlations in a Double Quantum Dot, Phys. Rev. Lett. 98, 056801 (2007).

[37] D. M. T. van Zanten, D. M. Basko, I. M. Khaymovich, J. P. Pekola, H. Courtois, and C. B. Winkelmann, Single Quantum Level Electron Turnstile, Phys. Rev. Lett. 116, 166801 (2016).

[38] M. Nahum, T. M. Eiles, and J. M. Martinis, Electronic microrefrigerator based on a normal insulator superconductor tunnel junction, Appl. Phys. Lett. 65, 3123 (1994).

[39] M. M. Leivo and J. P. Pekola, Efficient Peltier refrigeration by a pair of normal metal/insulator/superconductor junctions, Appl. Phys. Lett. 68, 1996 (1996).

[40] R. Sánchez, Correlation-induced refrigeration with superconducting single-electron transistors, Appl. Phys. Lett. 111, 223103 (2017).

[41] H. Hsu, M. Silveri, A. Gunyhó, J. Goetz, G. Catelani, and M. Möttönen, Tunable refrigerator for nonlinear quantum electric circuits, Phys. Rev. B 101, 235422 (2020).

[42] A. Martín-Rodero and A. Levy Yeyati, Josephson and Andreev transport through quantum dots, Adv. Phys. 60, 899 (2011).

[43] See Supplemental Material at http://link.aps.org/ supplemental/10.1103/PhysRevLett.125.247701 for further details on the theoretical methods, which includes Refs. [4452].

[44] H. Haug and A. P. Jauho, Quantum Kinetics in Transport and Optics of Semiconductors (Springer, New York, 2008).

[45] E. Vecino, A. Martín-Rodero, and A. Levy Yeyati, Josephson current through a correlated quantum level: Andreev states and $\pi$ junction behavior, Phys. Rev. B 68, 035105 (2003).

[46] Y. Yamada, Y. Tanaka, and N. Kawakami, Interplay of Kondo and superconducting correlations in the nonequilibrium Andreev transport through a quantum dot, Phys. Rev. B 84, 075484 (2011).

[47] J. Wang, B. Wang, W. Ren, and H. Guo, Conservation of spin current: Model including self-consistent spin-spin interaction, Phys. Rev. B 74, 155307 (2006).

[48] H. Bruus and K. Flensberg, Many-Body Quantum Theory in Condensed Matter Physics: An Introduction (Oxford University Press, Oxford, 2004).

[49] P. Trocha and J. Barnaś, Spin-polarized Andreev transport influenced by Coulomb repulsion through a two-quantumdot system, Phys. Rev. B 89, 245418 (2014).

[50] S. E. Nigg, R. P. Tiwari, S. Walter, and T. L. Schmidt, Detecting nonlocal Cooper pair entanglement by optical Bell inequality violation, Phys. Rev. B 91, 094516 (2015).

[51] R. C. Dynes, V. Narayanamurti, and J. P. Garno, Direct Measurement of Quasiparticle-Lifetime Broadening in a Strong-Coupled Superconductor, Phys. Rev. Lett. 41, 1509 (1978).

[52] J. P. Pekola, V. F. Maisi, S. Kafanov, N. Chekurov, A. Kemppinen, Yu. A. Pashkin, O.-P. Saira, M. Möttönen, and J.S. Tsai, Environment-Assisted Tunneling as an Origin of the Dynes Density of States, Phys. Rev. Lett. 105, 026803 (2010).

[53] O. Sauret, D. Feinberg, and T. Martin, Quantum master equations for the superconductor-quantum dot entangler, Phys. Rev. B 70, 245313 (2004).

[54] J. Eldridge, M. G. Pala, M. Governale, and J. König, Superconducting proximity effect in interacting doubledot systems, Phys. Rev. B 82, 184507 (2010).

[55] R. Sánchez, P. Burset, and A. Levy Yeyati, Cooling by Cooper pair splitting, Phys. Rev. B 98, 241414(R) (2018). 